\title{
Frequency and Time Domain Variability Analysis of an On-Chip Inverted Embedded Microstrip Line Using a Macromodeling-based Stochastic Galerkin Method
}

\author{
Dries Vande Ginste ${ }^{1}$, Daniël De Zutter ${ }^{1}$, Dirk Deschrijver ${ }^{1}$, Tom Dhaene ${ }^{1}$, Paolo Manfredi ${ }^{2}$, Flavio Canavero ${ }^{2}$ \\ ${ }^{1}$ Department of Information Technology, Ghent University, Sint-Pietersnieuwstraat 41, 9000 B-Gent, Belgium \\ E-mail: Dries.VandeGinste@UGent.be, Tel.: +3292643354 \\ 2 Dipartimento di Elettronica, Politecnico di Torino, Corso Duca degli Abruzzi 24, 10129 Torino, Italy
}

\begin{abstract}
In this contribution a novel stochastic Galerkin method is proposed to analyze the parameter variability of uniform onchip interconnects. This efficient and accurate stochastic modeling method is made possible, specifically for on-chip interconnects, by first constructing parameterized macromodels of the per unit length transmission line parameters. The theory is illustrated by means of a numerical example, i.e. an inverted embedded microstrip line, of which the variability is analyzed in both the frequency and the time domain. A comparison with a standard Monte Carlo technique validates the new approach.
\end{abstract}

\section{Introduction}

It is a well-known fact that manufacturing tolerances are playing a significant role during the design of on-chip interconnect structures. Increasing signaling speeds and further miniaturization only add to the problem. Therefore, to assess signal integrity behavior, circuit designers need to have access to powerful modeling tools that also allow predicting parameter variability effects in acceptable time.

Recent research efforts have led to a stochastic modeling technique, especially conceived to study parameter variability of uniform transmission lines, such as traces on boards [1] and cable harnesses [2]. To this end, a Polynomial Chaos (PC) expansion of the pertinent Telegrapher's equations in combination with a Galerkin weighting was put forward. This technique is called the Stochastic Galerkin Method (SGM) and has proven to be very effective for this kind of problem. Unfortunately, the technique is not readily extended to on-chip interconnect structures, as it makes use of basic numerical or heuristic models for the per unit length (p.u.l.) parameters of the transmission lines at hand, also assuming that these p.u.l. parameters are independent of frequency. For on-chip interconnects, in the presence of semiconductors, the computation of these p.u.l. parameters is not straightforward.

In [3], a simple but robust Monte Carlo (MC) analysis technique was implemented for an on-chip line, being an inverted embedded microstrip (IEM) line. The manufacturing process (etching or electrolytic growth) yields a line with a random trapezoidal cross-section, rather than a rectangular one. Via $\mathrm{MC}$ analysis, the effects on signal integrity of this randomness was studied. The MC approach was made tractable thanks to a two-step approach. First, the p.u.l. parameters of the IEM line were accurately computed using a two-dimensional (2-D) electromagnetic (EM) modeling technique, leading to a set of tabulated data. All high-frequency phenomena and loss mechanisms, leading to skin-effect, slow-wave effect, etc, were accounted for. Second, these tabulated p.u.l. parameters were converted into parameterized macromodels. Once these analytical, closed-form expressions are available, a rapid implementation of the MC runs is possible.

In this contribution, an SGM for the single IEM line is proposed. In contrast to previous work [1] and [2], an SGM analysis can be implemented by making use of parameterized macromodels of the p.u.l. parameters. Of course, as will be demonstrated here, this novel SGM largely outperforms the MC analysis of [3], both in terms of speed and accuracy. The effects on signal integrity of the randomness of the trapezoidal crosssection of the line are assessed. In this contribution, the focus is on a single line with a single stochastic parameter, studied in both frequency and time domain, whereas in [4] general multiconductor on-chip lines with multiple stochastic parameters are studied in frequency domain.

\section{Macromodeling based SGM for a single on-chip line}

Consider the following Telegrapher's equations for a single uniform transmission line:

$$
\begin{aligned}
\frac{d}{d z} V(z, s, \beta) & =-Z(s, \beta) I(z, s, \beta), \\
\frac{d}{d z} I(z, s, \beta) & =-Y(s, \beta) V(z, s, \beta),
\end{aligned}
$$

where $V(z, s, \beta)$ and $I(z, s, \beta)$ are the voltage and the current along the line, which are function of the distance $z$ along the line and the complex frequency $s=j 2 \pi f$. They also depend on the stochastic parameter $\beta$. This latter can be a material parameter or a geometrical parameter. In this contribution (see further), $\beta$ determines the shape of the cross-section of the line. Often, a normalization of the stochastic parameter is put forward as follows:

$$
\beta=\mu_{\beta}\left(1+\sigma_{\beta} \xi\right),
$$

where $\mu_{\beta}$ and $\sigma_{\beta}$ represent the mean and the normalized standard deviation of $\beta$, respectively, and with $\xi$ the corresponding normalized stochastic parameter. For example, in the case that $\beta$ is a Gaussian random variable (RV), $\xi$ has a standard normal distribution

$$
W_{\xi}(\xi)=\frac{1}{\sqrt{2 \pi}} e^{-\frac{1}{2} \xi^{2}} .
$$

The p.u.l. impedance and admittance, $Z(s, \beta)$ and $Y(s, \beta)$ respectively, are also frequency dependent and influenced by $\beta$. These quantities can be further decomposed into the wellknown p.u.l. resistance $R(s, \beta)$ (in $\Omega / \mathrm{m}$ ), inductance $L(s, \beta)$ (in $\mathrm{L} / \mathrm{m}$ ), conductance $G(s, \beta)$ (in $\mathrm{S} / \mathrm{m}$ ) and capacitance $C(s, \beta)$ (in $\mathrm{F} / \mathrm{m}$ ), as follows:

$$
\begin{aligned}
& Z(s, \beta)=R(s, \beta)+s L(s, \beta), \\
& Y(s, \beta)=G(s, \beta)+s C(s, \beta) .
\end{aligned}
$$


The two-step approach, proposed in [3], is applied. First, the p.u.l. parameters are very accurately computed using the 2-D EM solver presented in $[5,6]$. This solver starts from a quasiTM assumption of the fields, which is a valid assumption for the small interconnects under consideration. Making use of a Dirichlet-to-Neumann boundary operator and a careful formulation of the circuit currents in the presence of the semiconductors, the complex capacitance and complex inductance problems are cast as boundary integral equations (BIEs), taking into account all substrate loss mechanisms and the finite size, conductivity and shape of the metallic interconnect. Solving the BIEs leads to a set of tabulated RLGC-data, being a function of the frequency $f$ and of the stochastic parameter $\beta$. Second, the data samples so obtained are used as input for a macromodeling procedure. This results in the following closed-form expression for the p.u.l. impedance and admittance:

$$
X^{\mathrm{mm}}(s, \beta)=\sum_{v=1}^{V} w_{v} X^{\mathrm{umm}}\left(s, \beta_{v}\right) \prod_{\substack{k=1 \\ k \neq v}}^{V}\left(\beta-\beta_{k}\right),
$$

where $X$ stands for $Z$ and $Y$. The bivariate parameterized macromodels (7) are constructed by first keeping the parameter $\beta$ fixed, leading to a set of $V$ univariate rational macromodels $X^{\mathrm{umm}}\left(s, \beta_{v}\right)$, obtained via a Vector Fitting (VF) [7-9] procedure, and then stringing these rational models together by means of barycentric Lagrange interpolation [10]. The frequency-dependency can be accurately captured using the proposed rational modeling via VF; the variation of the p.u.l. parameters w.r.t. $\beta$ is usually rather smooth, hence, a careful choice of the barycentric weights $w_{v}$ leads to a polynomial interpolation (7).

At this point, the procedure starts to differ from the one presented in [3]. Instead of solving the Telegrapher's equations (1) and (2) for many samples of the parameter $\beta$, drawn according to its stochastic distribution, as prescribed by MC, an intrusive stochastic solution method is now applied. In this procedure, first, the known p.u.l. parameters and the unknown voltage and current are expanded into a set of $K+1$ basis functions $\left\{\phi_{k}\right\}_{k=0}^{K}$, as follows:

$$
\begin{aligned}
X^{\mathrm{mm}}(s, \beta) & =\sum_{k=0}^{K} X_{k}(s) \phi_{k}(\xi), \\
V(z, s, \beta) & =\sum_{k=0}^{K} V_{k}(z, s) \phi_{k}(\xi), \\
I(z, s, \beta) & =\sum_{k=0}^{K} I_{k}(z, s) \phi_{k}(\xi) .
\end{aligned}
$$

The basis functions are orthogonal polynomials, chosen according to the Wiener-Askey scheme [11]. For example, for the case of a Gaussian RV, $\left\{\phi_{k}\right\}_{k=0}^{K}$ is a set of Hermite polynomials, and their orthogonality is expressed using the proper inner product and w.r.t. the weighting function (4):

$$
<\phi_{k}(\xi), \phi_{m}(\xi)>=\int_{-\infty}^{+\infty} \phi_{k}(\xi) \phi_{m}(\xi) W_{\xi}(\xi) d \xi=m ! \delta_{k m}
$$

where $\delta_{k m}$ is the Kronecker delta. Given this orthogonality, the $(K+1)$ expansion coefficients of the known p.u.l. parameters are given by:

$$
X_{k}(s)=\frac{<X^{\mathrm{mm}}(s, \beta), \phi_{k}(\xi)>}{k !}, k=0, \ldots, K .
$$

Thanks to the macromodeling procedure, these numbers $X_{k}(s)$ can now be calculated very efficiently and accurately, as numerical integration schemes are no longer required. Substitution of (8), (9) and (10) into (1) and (2), yields a set of $2(K+1)$ coupled differential equations in the $2(K+1)$ unknown expansion coefficients $V_{k}(z, s)$ and $I_{k}(z, s)$ for the voltages and the currents along the line, respectively. These new equations are still dependent on the stochastic parameter $\beta$, or rather, on its normalized counterpart $\xi$. This dependency is now removed by Galerkin weighting of these equations, using the same set of test functions $\left\{\phi_{m}\right\}_{m=0}^{K}$, yielding the following result:

$$
\begin{aligned}
& \frac{d}{d z} V_{m}(z, s)=-\sum_{k=0}^{K} \sum_{l=0}^{K} \alpha_{k l m} Z_{k}(s) I_{l}(z, s), \\
& \frac{d}{d z} I_{m}(z, s)=-\sum_{k=0}^{K} \sum_{l=0}^{K} \alpha_{k l m} Y_{k}(s) V_{l}(z, s),
\end{aligned}
$$

with

$$
\alpha_{k l m}=<\phi_{k}(\xi) \phi_{l}(\xi), \phi_{m}(\xi)>/ m !,
$$

for all $m=0, \ldots, K$. The equations (13) and (14) represent a matrix ordinary differential equation (ODE) in the $2(K+1)$ unknown expansion coefficients for the voltage and the current, which no longer depends on $\xi$. After introduction of the proper boundary conditions, as explained in [1], this ODE can be solved using standard techniques. The final solution is obtained by substituting the expansion coefficients $V_{k}(z, s)$ and $I_{k}(z, s)$, $k=0, \ldots, K$, back into (9) and (10), yielding closed-form expressions for the voltages and currents of the lines as function of the position $z$, the complex frequency $s$, and the $\operatorname{RV} \beta$, for which a pertinent distribution, e.g. Gaussian, was assumed. From this result, stochastic moments or functions can be computed, using standard techniques [12]. This is demonstrated in the next section.

\section{Results}

We consider the IEM line of [3], and compare the MC approach with the new SGM of the previous section. The IEM line has a cross-section as shown in Fig. 1. This particular interconnect is embedded in a layered substrate consisting of a $30 \mu \mathrm{m}$ thick doped silicon substrate, with a relative permittivity of 11.7 and a conductivity of $10 \mathrm{~S} / \mathrm{m}$, and a $11.4 \mu \mathrm{m}$ thick $\mathrm{SiO}_{2}$ insulator, with a relative permittivity of 3.9 and loss tangent of 0.001. The ground plane is placed on top of the layered substrate and it is made out of $3 \mu \mathrm{m}$ thick Aluminum with a conductivity of $3.77 \cdot 10^{7} \mathrm{~S} / \mathrm{m}$. The signal conductor is found at a height of $6.4 \mu \mathrm{m}$ above the semiconductor-insulator interface. It is made out of $2 \mu \mathrm{m}$ thick Aluminum. Whereas the top side of this line's cross-section is fixed to $2 \mu \mathrm{m}$, the base $\beta$ is considered to be random. The random trapezoidal shape, so 


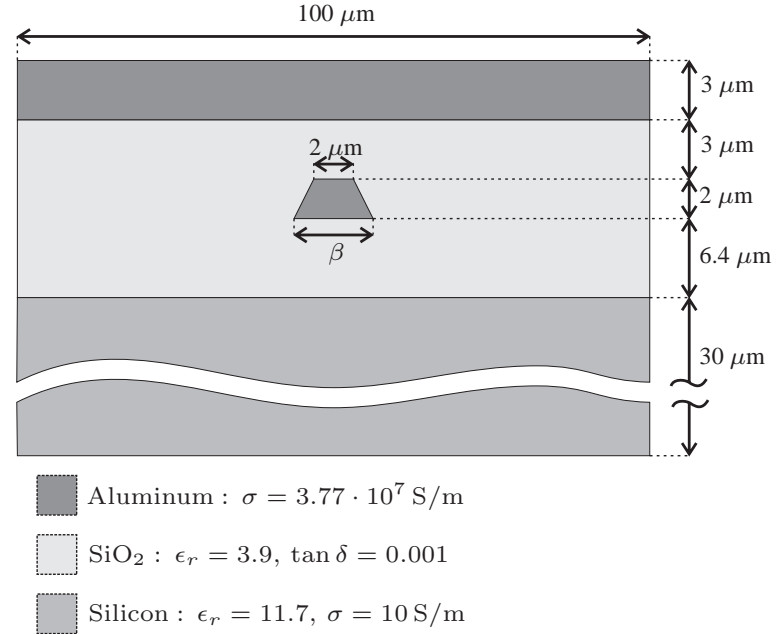

Figure 1: Cross-section of the IEM line (not on scale).

obtained, is induced by the manufacturing process (etching or electrolytic growth). In the remainder of this contribution it is assumed that $\beta$ is a Gaussian RV with a mean $\mu_{\beta}=2 \mu \mathrm{m}$ and a normalized standard deviation $\sigma_{\beta}=10 \%$. With this IEM line a source-line-load configuration is now constructed. The line is given a length of $\mathcal{L}=1 \mathrm{~mm}$. At its near end a lowimpedance voltage source, with an internal impedance of $1 \Omega$, is connected. At the far end, the line is terminated by a capacitive load $Z_{L}=\left(s C_{L}+1 / R_{L}\right)^{-1}$, with $C_{L}=1 \mathrm{pF}$ and $R_{L}=1 \mathrm{k} \Omega$.

In the frequency domain, using a voltage source of $1 \mathrm{~V}$, the variability of the output voltage $V_{L}(s, \beta) \equiv V(z=\mathcal{L}, s, \beta)$ at the load is studied over a broad frequency range up to $100 \mathrm{GHz}$ (Fig. 2(a)). In Fig. 2(b) there is zoomed in on the resonance around $7 \mathrm{GHz}$. The full black line indicates the mean $\mu_{\left|V_{L}\right|}$ of this output characteristic and the dashed lines show the $\pm 3 \sigma_{\left|V_{L}\right|}$ deviations from this mean $\mu_{\left|V_{L}\right|}$, both computed using the novel SGM technique. These results are compared to the ones of [3], where an MC run was performed using 50000 samples of the $\mathrm{RV} \beta$, drawn according to the appropriate Gaussian distribution. The gray lines on Fig. 2 correspond to 100 samples of this MC run; the circles (०) and squares (口) indicate the mean $\mu_{|H|}$ and the $\pm 3 \sigma_{|H|}$ deviations, respectively, computed using the 50000 samples. (For clarity, the circles and squares are not shown on Fig. 2(a).) It can be observed that the novel technique and the MC run are in perfect agreement. The efficiency of the SGM is however much higher. On a standard laptop, the analysis leading to Fig. 2 is completed in $0.13 \mathrm{~s}$ when leveraging the SGM, while $4.13 \mathrm{~s}$ are needed for the MC run. So, a significant speed-up factor of 32 is realized. (Both the SGM and the MC analysis rely on the same macromodels of the p.u.l. parameters, which are constructed offline. Including all necessary 2-D EM simulations, this takes about $140 \mathrm{~s}$ on a standard laptop.) Apart from comparing stochastic moments, also, complete stochastic functions, such as the probability density function (PDF) and the cumulative distribution function (CDF) can be computed. This is demontrated in Fig. 3, where the PDF and CDF of the magnitude of the output voltage are shown at $7 \mathrm{GHz}$. Comparing the SGM with MC again leads to the conclusion that excellent accuracy is obtained.

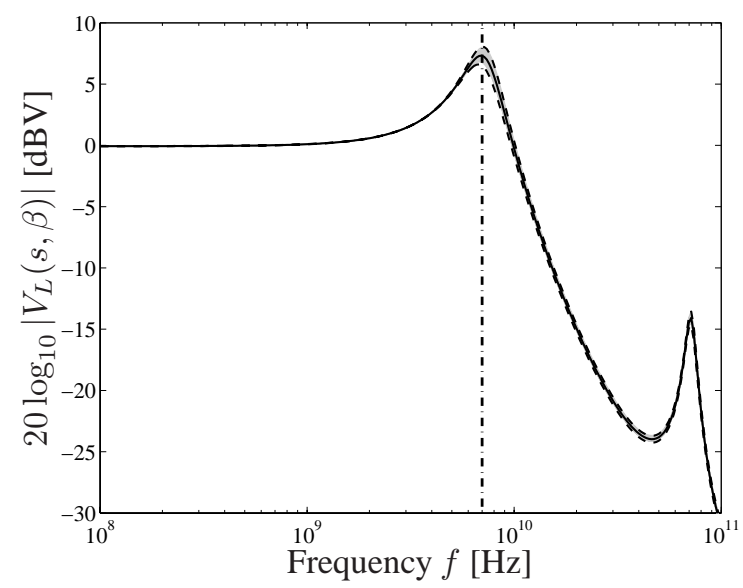

(a) Response as a function of frequency

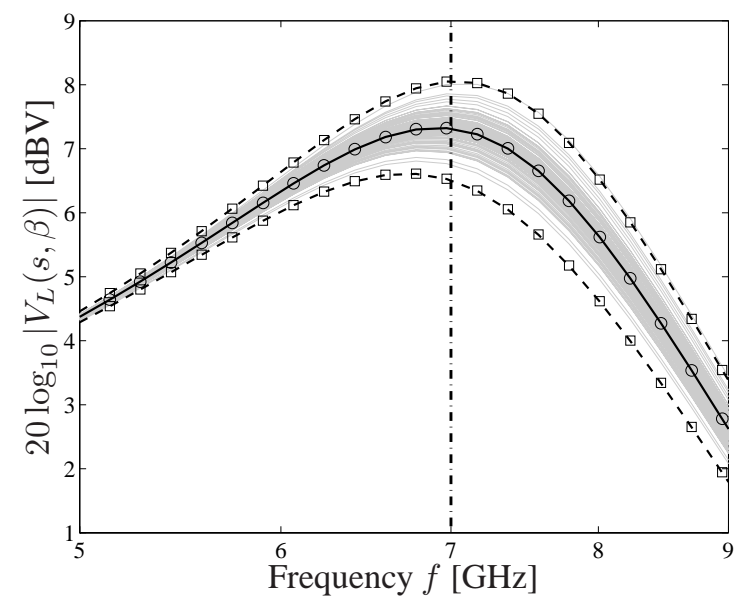

(b) Detail of Fig. 2(a) around $7 \mathrm{GHz}$, clearly illustrating the effect of the stochastic parameter $\beta$

Figure 2: Bode plot of the magnitude of the output voltage $V_{L}(s, \beta)$ for the single IEM line. Full black line: mean $\mu_{\left|V_{L}\right|}$ computed using the novel technique; Dashed black line: $\pm 3 \sigma_{\left|V_{L}\right|}$-variations computed using the novel technique; Gray lines: 100 samples from the MC run; Circles (०): mean $\mu_{\left|V_{L}\right|}$ computed using MC technique; Squares ( $\square$ ): $\pm 3 \sigma_{\left|V_{L}\right|}$-variations computed using $\mathrm{MC}$ technique.

After a post-processing step and in the presence of linear terminations, the frequency-domain results can be used to perform a transient analysis. We use the same source-line-load configuration as described above, but now the voltage source produces a ramped step, going from $0 \mathrm{~V}$ to $1 \mathrm{~V}$ in a rise time of $50 \mathrm{ps}$. So, the voltage $V_{L}(t)$ at the load becomes a function of the time $t$ and we compute the maximum voltage overshoot at the load, i.e. $\max _{t>0}\left(V_{L}(t)\right)$. The PDF and CDF of this quantity are shown in Fig. 4, again validating our new approach. Figures as Fig. 4 also provide valuable information for interconnect designers.

\section{Conclusions}

In this contribution a novel stochastic modeling procedure to study the influence of parameter variability on the signal integrity of an on-chip interconnect was outlined. First, the p.u.l. parameters of the line need to be accurately computed. 


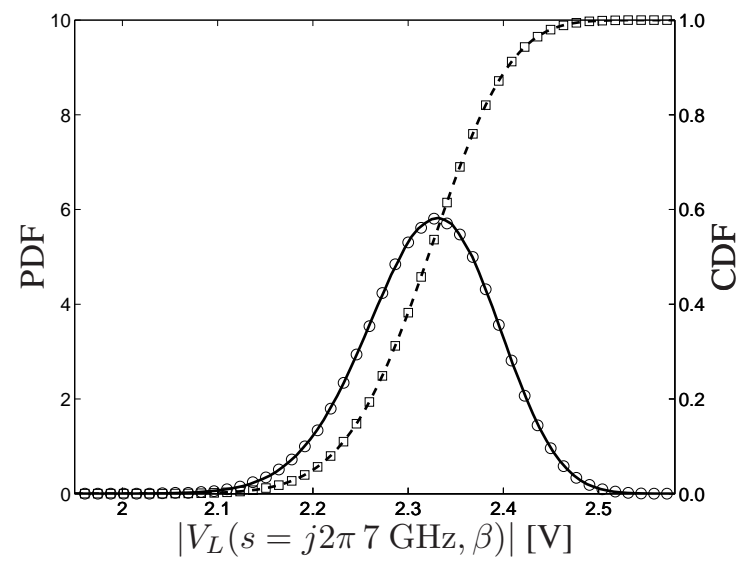

Figure 3: PDF and CDF of the magnitude of output voltage $V_{L}(s, \beta)$ at $7 \mathrm{GHz}$ for the single IEM line. Full black line: PDF computed using the novel technique; Dashed black line: CDF computed using the novel technique; Circles (o): PDF computed using the MC technique; Squares ( $\square$ ): CDF computed using the MC technique.

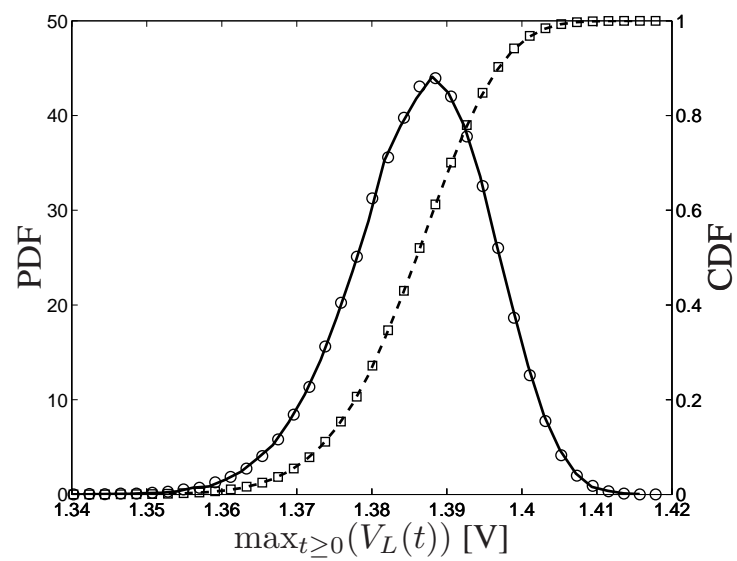

Figure 4: PDF and CDF of the overshoot, i.e. the maximum value of $V_{L}(t)$. Full black line: PDF computed using the novel technique; Dashed black line: CDF computed using the novel technique; Circles (०): PDF computed using the MC technique;

Squares $(\square)$ : CDF computed using the MC technique.

To this end, a two-step approach was used, combining very precise 2-D EM modeling with parameterized macromodeling, leading to accurate, closed-form expressions of the p.u.l. parameters. This is necessary, as for on-chip lines, no simple numerical schemes or heuristic formulas are available to compute the p.u.l. parameters. Next, relying on these parameterized macromodels, an SGM is implemented by applying a PC expansion of the Telegrapher's equations and then weighting these expanded equations by means of a Galerkin procedure. Finally, the voltage and current along the interconnect are known, being a function of the stochastic parameter under consideration. The novel formalism has been applied to a single IEM line with a random trapezoidal cross-section, in order to analyze its signal integrity behavior. Compared to an MC analysis, the new technique shows excellent accuracy and a considerably improved efficiency.

\section{References}

[1] I. S. Stievano, P. Manfredi, and F. G. Canavero, "Parameters variability effects on multiconductor interconnects via hermite polynomial chaos," IEEE Trans. on Components, Packaging and Manufacturing Technology, vol. 1, no. 8, pp. 1234-1239, Aug. 2011.

[2] —-, "Stochastic analysis of multiconductor cables and interconnects," IEEE Trans. Electromagn. Compat., vol. 53, no. 2, pp. 501-507, May 2011.

[3] D. Vande Ginste, D. De Zutter, D. Deschrijver, T. Dhaene, and F. Canavero, "Macromodeling based variability analysis of an inverted embedded microstrip line," in Proc. of the 20th IEEE Int. Conf. on Electrical Performance of Electronic Packaging and Systems (EPEPS), San Jose, CA, USA, 23 - 26 Oct. 2011, pp. 153-156.

[4] D. Vande Ginste, D. De Zutter, D. Deschrijver, T. Dhaene, P. Manfredi, and F. Canavero, "Stochastic modeling based variability analysis of on-chip interconnects," Submitted to IEEE Transactions on Components, Packaging and Manufacturing Technology, Dec. 2011.

[5] T. Demeester and D. De Zutter, "Quasi-TM transmission line parameters of coupled lossy lines based on the Dirichlet to Neumann boundary operator," IEEE Trans. Microw. Theory Tech., vol. 56, no. 7, pp. 1649-1660, Jul. 2008.

[6] T. Demeester, D. Vande Ginste, and D. De Zutter, "Accurate study of the electromagnetic and circuit behavior of finite conducting wedges and interconnects with arbitrary cross-sections," in Proc. of 19th IEEE Int. Conf. on Electrical Performance of Electronic Packaging and Systems (EPEPS), Austin, TX, USA, 24 - 27 Oct. 2010, pp. 133-136.

[7] B. Gustavsen and A. Semlyen, "Rational approximation of frequency domain responses by vector fitting," IEEE Trans. Power Del., vol. 14, no. 3, pp. 1052-1061, Jul. 1999.

[8] B. Gustavsen, "Improving the pole relocating properties of vector fitting," IEEE Trans. Power Del., vol. 21, no. 3, pp. 1587-1592, Jul. 2006.

[9] D. Deschrijver, M. Mrozowski, T. Dhaene, and D. De Zutter, "Macromodeling of multiport systems using a fast implementation of the vector fitting method," IEEE Microwave and Wireless Components Letters, vol. 18, no. 6, pp. 383-385, Jun. 2008.

[10] J. P. Berrut and L. N. Trefethen, "Barycentric Lagrange interpolation," SIAM Review, vol. 46, no. 3, pp. 501-517, Sep. 2004.

[11] D. Xiu and G. E. Karniadakis, "The Wiener-Askey polynomial chaos for stochastic differential equations," SIAM J. Sci. Comput., vol. 24, no. 2, pp. 619-644, 2002.

[12] A. Papoulis, Probability, Random Variables, and Stochastic Processes, 3rd ed. McGraw-Hill, 1991. 